\title{
Les vantaux de la façade occidentale de la cathédrale Saint-Étienne d'Auxerre (Yonne)
}

Sylvain Aumard

\section{(2) OpenEdition}

1 Journals

\section{Édition électronique}

URL : https://journals.openedition.org/cem/11306

DOI : 10.4000/cem.11306

ISSN : 1954-3093

Éditeur

Centre d'études médiévales Saint-Germain d'Auxerre

\section{Édition imprimée}

Date de publication : 15 août 2009

Pagination : 93-96

ISSN : 1623-5770

\section{Référence électronique}

Sylvain Aumard, «Les vantaux de la façade occidentale de la cathédrale Saint-Étienne d'Auxerre

(Yonne) ", Bulletin du centre d'études médiévales d'Auxerre | BUCEMA [En ligne], 13 | 2009, mis en ligne le 23 septembre 2009, consulté le 22 septembre 2022. URL : http://journals.openedition.org/cem/11306 ; DOI : https://doi.org/10.4000/cem.11306

Ce document a été généré automatiquement le 22 septembre 2022.

\section{(c) (i) (5)}

Creative Commons - Attribution - Pas d'Utilisation Commerciale - Partage dans les Mêmes Conditions 4.0 International - CC BY-NC-SA 4.0

https://creativecommons.org/licenses/by-nc-sa/4.0/ 


\title{
Les vantaux de la façade occidentale de la cathédrale Saint-Étienne d'Auxerre (Yonne)
}

\author{
Sylvain Aumard
}

1 L'année 2008 a été celle de l'achèvement des travaux de restauration et, par voie de conséquence, celle des ultimes observations archéologiques ${ }^{1}$. Ces dernières ont porté sur les vantaux de la façade occidentale et avaient été initiées, quelques années auparavant, par l'équipe de Stuttgart, qui avait amorcé des relevés au niveau du portail central. Le caractère novateur de cette opération s'inscrit dans la lignée de la démarche impulsée dès le début de ce vaste projet par le conseil scientifique et consistant à soumettre à l'interrogation archéologique chaque composante du monument - maçonneries, charpentes, couvertures, verrières... L'originalité de cette étude tient notamment au fait que les vantaux de ce monument n'ont pratiquement jamais fait l'objet de véritables analyses à l'égal du bâti ; au mieux, ces ouvrages font l'objet de relevés de principe, parfois très détaillés, mais la lecture de leur histoire depuis leur origine n'est jamais entreprise (repentirs, réparations...). Dans le cas de la cathédrale d'Auxerre, cette démarche a bénéficié d'une double opportunité conjoncturelle :

2 - bénéficier des travaux de restauration pour accéder à de nouvelles données et tester l'application des méthodes de l'archéologie du bâti à ce type de contexte ;

3 - profiter de la préparation de la réédition (augmentée) d'un des albums du Centre de recherches sur les monuments 
historiques (CRMH, ministère de la Culture) pour diffuser les résultats ${ }^{2}$.

L'intervention du CEM avait pour objectif de procéder à un véritable relevé archéologique détaillé des structures du portail nord - en ayant notamment recourt aux nouvelles technologies de numérisation en $3 D^{3}-$ et de faire dater ses bois. D'après l'étude de faisabilité de l'été $2006{ }^{4}$, la dendrochronologie pouvait être réalisée par lecture directe des cernes sur les pièces de bois, sans prélèvement de matière et son accomplissement pouvait être facilité, ici, par le solide référentiel dendrochronologique constitué lors des études précédentes ${ }^{5}$. Le choix de concentrer les efforts sur le portail nord a été guidé à la fois par le potentiel de datation - accessibilités des abouts de montants et de panneaux - et par l'absence de tambour offrant, à l'inverse du portail sud, le recul nécessaire à l'accomplissement des travaux de topographie. En outre, le CRMH s'est chargé de l'achèvement des relevés des vantaux du portail central. Comme il s'agissait d'un terrain nouveau pour notre équipe, la collaboration avec ce centre a largement été étendue, en associant aussi bien d'autres scientifiques et restaurateurs (D. Pousset, C. Locatelli et $T$. Palanque), que des artisans particulièrement qualifiés dans le domaine (É. Leriche et M. Mignot).

5 En attendant la présentation détaillée des données dans la future publication du CRMH, on peut résumer les apports de cette étude aux points suivants. Le portail nord est constitué de trois registres de panneaux insérés entre des traverses et des montants intermédiaires par des assemblages en rainure et languette. Les restaurations anciennes concernent essentiellement les parties inférieures et, d'une manière générale, elles n'ont pas modifié l'aspect de l'ouvrage à l'exception de la mouluration externe des traverses intermédiaires supérieures complètement ravalée. La principale incohérence concerne le système de rotation assuré actuellement par des gonds et des pentures. Celui-ci est en effet désaxé par rapport à un premier système rapidement abandonné et constitué d'un pivot sur platine en fer. La conception de l'ouvrage fait une large place au métal au travers d'une multitude de pièces de serrurerie, destinée à renforcer les assemblages au dimensionnement trop faible. La dendrochronologie permet d'envisager une réalisation vers la fin du $X^{e}$ siècle et/ou le début du XVI ${ }^{e}$ siècle, plus tardive que celle des vantaux du portail central suggérée par les textes (vers 1500 environ). Dans 
tous les cas, ces données confirment notre hypothèse d'une finalisation tardive du portail nord argumentée sur la base des nombreuses incohérences que recèlent ses dispositions ${ }^{6}$. Le portail sud n'a pu être aussi finement étudié en raison de la présence d'un tambour formant un sas d'accès. Toutefois, l'étude des traces médiévales de l'oratoire Notre-Dame-des-Vertus permet de proposer comme hypothèse de chronologie, soit le XIII siècle (avant édification de l'oratoire), soit le $X V I^{\mathrm{e}}$ siècle (après démolition de l'oratoire) ${ }^{7}$. De futures datations par radiocarbone sur les cernes périphériques des deux montants-pivots devraient permettre de trancher dans un proche avenir.

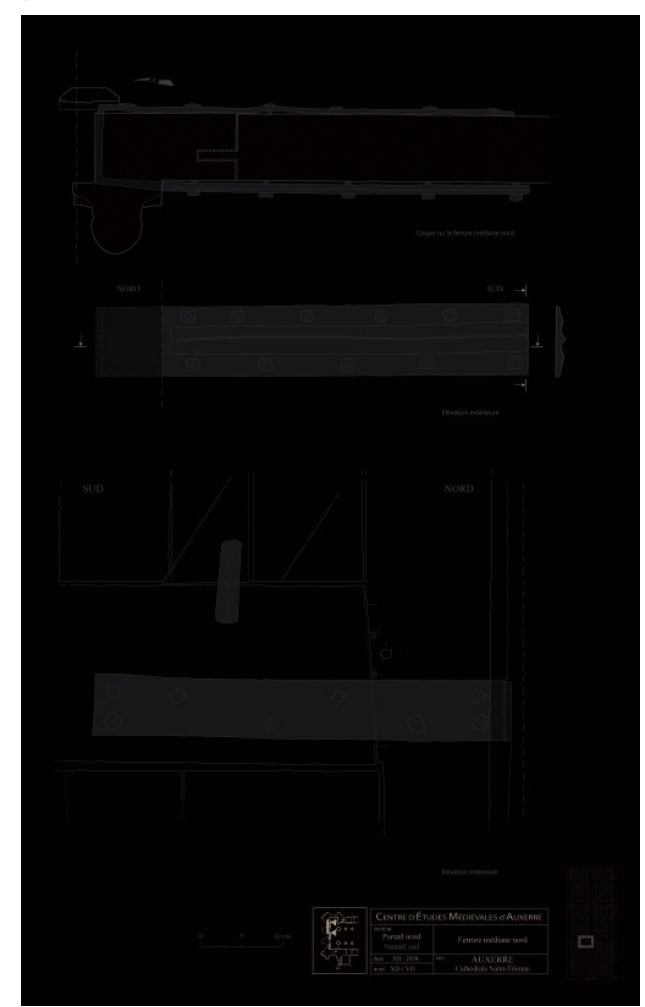

Fig. 1 - Auxerre, cathédrale Saint-Étienne, vantaux du portail nord de la façade occidentale assemblage à tenon et mortaise renforcé par une bande de fer en « U » (CEM, Auxerre, X. D'aire et V. Hontcharenko).

\section{NOTES}

1. Avec les collaborations de : Fabrice Henrion, Vanessa Hontcharenko et Xavier D’aire (CEM, Auxerre); Rachel Touzé et Christel Guillot 
(Centre de recherche sur les monuments historiques/ministère de la Culture, Paris); Thierry Palanque (LP3, Semur-en-Auxois); Didier Pousset et Christine Locatelli (Dendrochronology Consulting, SaintVit) ; Étienne Leriche (menuisier, Auxerre); M. Mignot (Ets Perrault, Angers) ; TPLM3D (Chassieu).

2. À paraître aux Éditions du Patrimoine (Monum).

3. Prestation réalisée par l'entreprise TPLM3D (Chassieu).

4. Étude réalisée par D. Pousset et financée par le CEM en 2006.

5. Ce référentiel de plus de 250 prélèvements faits de la cathédrale d'Auxerre une référence unique en Europe dans le domaine de l'étude des bois médiévaux. Cette particularité a été présentée au colloque international de dendrochronologie tenu à Pékin en octobre 2005.

6. Cf. Bulletin du Centre d'études médiévales d'Auxerre, 2008, 12, p. 117-121 [http://cem.revues.org/document6212.html].

7. Cf. Bulletin du Centre d'études médiévales d'Auxerre, 2008, 12, p. 113-117 [http://cem.revues.org/document6212.html].

INDEX

Index géographique : France/Auxerre

Mots-clés : cathédrale, vantail 